\title{
Temporal primacy overrides prior training in serial compound conditioning of the rabbit's nictitating membrane response
}

\author{
E. JAMES KEHOE, BERNARD G. SCHREURS, and PEITA GRAHAM \\ University of New South Wales, Kensington, New South Wales, Australia
}

\begin{abstract}
Conditioning of the rabbit's nictitating membrane response was conducted with a serial compound CSA-CSB-US. In the present experiments, prior training of CSB was pitted against the temporal primacy of CSA. Prior training of CSB was able to only weakly block CR acquisition to the added CSA, but CSA caused a pronounced decline in responding to the pretrained CSB. By the end of training, high levels of responding were sustained only in the final portion of the serial compound in which CSA or its traces coincided with CSB. These results provide support for real-time models as exemplified by Sutton and Barto (1981).
\end{abstract}

The present experiments examined the acquisition of the rabbit's nictitating membrane (NM) response to the components of a serial compound stimulus (CSA-CSBUS) after previous reinforced training with CSB. Empirically, these experiments were designed to pit the prior training of CSB against the temporal primacy of CSA, both of which can impair conditioned response (CR) acquisition to components of a compound stimulus. That is to say, prior training of CSB can cause blocking of CR acquisition to CSA during subsequent serial compound training (e.g., Gibbs, 1979; Kohler \& Ayres, 1982). Conversely, the temporal primacy of CSA relative to CSB can cause deficits in CR acquisition to CSB (e.g., Egger \& Miller, 1962; Kehoe, 1979, 1983; Wickens, 1959, $1965,1973)$. Although blocking and temporal primacy effects have been widely studied in isolation, little is known about their interrelationships (see Kehoe, 1982, pp. 41-42).

At a theoretical level, the present experiments were intended to test the Sutton-Barto model of classical conditioning (Barto \& Sutton, 1982; Sutton \& Barto, 1981). In brief, the Sutton-Barto model can be viewed as a "realtime" implementation of Rescorla and Wagner's (1972) model. Like the Rescorla-Wagner model, the Sutton-Barto model assumes that two compounded stimuli will compete for a fixed amount of associative strength that can be maintained at any one time by the unconditioned stimulus (US). Accordingly, blocking occurs, because prior training with one component permits it to capture all the available associative strength, thus preventing an added component from gaining any increments in associative strength. Where the Sutton-Barto model differs from the Rescorla-Wagner model is in the treatment of real-time

This research was supported by Grant A28315236 from the Australian Research Grants Committee. The authors thank J. W. Moore for his aid in preparing this article. Correspondence should be sent to $E$. J. Kehoe, School of Psychology, University of New South Wales, Kensington, NSW 2033, Australia. processes that operate throughout the presentation of a conditioned stimulus (CS). The Rescorla-Wagner model assumes that associative strengths are altered only at the time of reinforcement, whereas the Sutton-Barto model assumes that associative strengths are evaluated and altered on a continuous basis prior to, during, and after the time of reinforcement. It is the elaboration of these realtime processes that enable the Sutton-Barto model to explain the deleterious effect of CSA's temporal primacy on CR acquisition to CSB

Sutton and Barto (1981) postulate that, at each moment $(t)$, the subject generates a response-output signal [si $(t)]$, which is the sum of the efficacies of concurrent stimuli; these include each CS's momentary associative strength [Vi(t)] and, when appropriate, the US's intrinsic responseevoking capacity. The subject also compares the current output signal with a sliding average of previous output signals [si $(t-1)]$. Changes in Vi from one moment to the next are computed in much the same way as in the Rescorla-Wagner model:

$$
V i(t+1)=V i(t)+c x i(t)[s i(t)-s i(t-1)]
$$

where $c=\mathrm{a}$ constant rate parameter $(0<c<1)$; $x i(t)=$ a "stimulus trace" variable that denotes the current eligibility of a $V i$ for modification $(0<x i(t)<1)$ (although $x i(t)$ grows and decays in a time-dependent manner following $\mathrm{CS}_{i}$ onset, the momentary value of $x i(t)$ corresponds to the CS salience parameter [oi] in the RescorlaWagner model); $s i(t)=$ the sum of the currently eligible associative strengths $[\Sigma V i(t)]$ plus the intrinsic capacity of the US when it occurs (because this sum is bounded to remain $\leq 1$, si, at the time of US presentation, corresponds to the asymptotic parameter [ $\lambda]$ in the RescorlaWagner model); and $s i(t-1)=$ the weighted sum of previous input efficacies (at the time of US presentation, $s i(t-1)$ corresponds to the sum of the current associative strengths $(\Sigma V i)$ in the Rescorla-Wagner model.

In summary, changes in associative strength of a CS arise whenever there is a discrepancy between the cur- 
rent output of the system [si(t)] and its recent output [si $(t-1)]$.

According to the Sutton-Barto model, the temporal primacy effect arises, perhaps surprisingly, from the same mechanism that causes second-order conditioning between CSA and CSB in a serial compound. Specifically, both the temporal primacy effect and second-order conditioning presumably result from a discrepancy between the current and recent output signals that can occur at the point of CSB onset. At CSB onset, CSA's associative strength (VA) would gain an increment from any associative strength previously gained by CSB (VB). Thus, as quickly as CSB gained associative strength, it would be transmitted to CSA. At the point of US onset, both CSA and CSB would gain associative strength on a competitive basis. Thus, VA could increase at the onsets of both CSB and the US, whereas VB would increase only at the onset of the US. (VB itself does not become eligible for change until some time after CSB onset. This lag in eligibility prevents VB from changing on the basis of its own value.) As VA approaches the asymptotic value, the summated associative strengths (VA + VB) could exceed that sustainable by the US. Thus, at US onset, there could be a negative discrepancy that would produce decrements in both VA and VB. On the next trial, VA would regain its lost value at CSB onset, while VB would continue to suffer decrements in associative strength at US onset until VA + VB stabilize at 1.00. Consequently, acquisition of associative strength by CSB could be effectively impaired and even reversed by CSA.

Computer simulations predict that the temporal primacy effect can emerge even when CSB has been given prior training (Barto \& Sutton, 1982, p. 227; Sutton \& Barto, 1981 , p. 151). Where CSA overlaps CSB, previously established responding to CSB should decline as CRs are acquired to CSA. However, where CSA terminates at CSB onset, CSA's representation will have largely decayed by the time of US onset and thus would offer relatively little competition to CSB. Consequently, in strictly sequential presentations of CSA and CSB, responding to CSB should be maintained, and a facilitation of CR acquisition to CSA should occur via second-order conditioning (Barto \& Sutton, 1982, p. 230).

To date, there have been no systematic tests of the Sutton-Barto model. The available examinations of serial compound conditioning after CSB pretraining have used exclusively sequential compounds, in which CSA terminates either prior to or at CSB onset. Their results provide some evidence favorable to the Sutton-Barto model. Specifically, Gibbs (1979, Experiment 2) examined acquisition of the rabbit NM response using a sequential compound in which a 400-msec CSA was terminated $1,000 \mathrm{msec}$ before a 400 -msec CSB. He found that after initial CSB training, responding to CSB was maintained throughout eight sessions of serial compound training. CR acquisition to CSA was facilitated, as compared with CR acquisition of an "uncoupled control" that received separate CSA-US and CSB-US trials, which prevented any second-order conditioning via CSA-CSB pairings. Nevertheless, some blocking of CSA did appear, because the facilitation of CR acquisition to CSA was less than that seen in a control group that did not receive initial CSB-US training. Similar results were obtained by Kehoe, Schreurs, and Amodei (1981, Experiment 2) who used a sequential compound in which a 400-msec CSA directly preceded a 400-msec CSB. After initial CSB-US training, high levels of responding to CSB were maintained throughout six sessions of serial compound training. CR acquisition to CSA showed only transitory and nonsignificant impairment relative to that of a control that did not receive initial CSB-US training. Elsewhere, partial blocking of response acquisition to CSA has been reported in taste aversion learning (Revusky, 1971, pp. 197-203), odor aversion learning (Cheatle \& Rudy, 1978), and conditioned suppression (Kohler \& Ayres, 1982; Pearce, Nicholas, \& Dickinson, 1981), but not in conditioned activity (Holland, 1980). Levels of responding to CSB have been largely unreported.

\section{EXPERIMENT 1}

Experiment 1 was conducted to test the Sutton-Barto model (1) by comparing serial compounds of overlapping versus sequential components, and (2) by extending serial compound training over a larger number of days than used in previous NM response studies to give more time for any temporal primacy effect to evidence itself. The temporal relations among components were arranged so as to maximize any difference between the overlapping and sequential compounds. In the overlapping compound, the duration of CSA was $2,000 \mathrm{msec}$, extending from the onset of CSA through a 500-msec CSB to coterminate with CSB at US onset. In the sequential compound, CSA was only $500 \mathrm{msec}$ in duration, leaving a 1,000 -msec trace interval between the offset of CSA and the onset of CSB.

\section{Method}

Subjects. The subjects were 32 naive female albino rabbits (Oryctolagus cuniculus) from the university's animal breeding and holding unit. On arrival, each was 70-80 days old and weighed approximately $1.5 \mathrm{~kg}$. The animals had free access to food and water in their home cages.

Apparatus. The apparatus and recording procedure for the NM response were patterned after those of Gormezano (1966; see Kehoe, 1986). The CSs were (1) a 1000-Hz, 85-dB (SPL) tone superimposed on an ambient noise level of $81 \mathrm{~dB}$, and (2) a 20-Hz flashing of the houselight. The US was a 50-msec, 3-mA, 50- $\mathrm{Hz}$ ac electric current delivered via stainless steel Autoclip wound clips positioned $10 \mathrm{~mm}$ apart and $15 \mathrm{~mm}$ posterior to the dorsal canthus of the right eye. The sequence and timing of stimulus events were controlled by an Apple II computer equipped with interfaces and software developed by Scandrett and Gormezano (1980). To monitor movements of the NM, a small, tinned copper wire hook was attached to a silk loop sutured in the NM of the rabbit's right eye. The other end of the hook contained a loop that fitted over the end of an L-shaped piano-wire lever that operated a photoelectric transducer. The signal from the transducer was amplified and transmitted to an analog/digital converter installed in the computer. No straps were used to restrain the external eyelids. 
Procedure. All rabbits received 1 day of preparation, 2 days of recovery, 1 day of adaptation, 4 days of Stage 1, CSB acquisition, training and 12 days of Stage 2, serial compound, training. On the preparation day, hair surrounding the rabbit's right eye was removed, a small loop of silk (000 Dynex) was sutured into the NM, and the animals were returned to their home cages for 2 days of recovery. On the adaptation day, the animals were placed in the conditioning apparatus for $70 \mathrm{~min}$, but neither a CS nor a US was presented.

Following adaptation, the animals were assigned randomly to one of four groups $(n=8)$ labeled B-OL, B-SQ, R-OL, and R-SQ. In Stage 1, Groups B-OL and B-SQ received 4 days of CSB-US training, while Groups R-OL and R-SQ were restrained in the experimental chambers. For Groups B-OL and B-SQ, each day of Stage 1 training contained $60 \mathrm{CSB}$-US trials in which both the CSB duration and CSB-US interval were $500 \mathrm{msec}$, values that remained the same during Stage 2. In Stage 2, Groups B-OL and R-OL received training with an overlapping compound (OL) in which the onset of CSA preceded the onset of CSB but overlapped and coterminated with CSB. In the overlapping compound, the CSA duration and CSA-US interval were $2,000 \mathrm{msec}$. In contrast, Groups B-SQ and $R-S Q$ received training with a sequential compound $(S Q)$, in which the CSA-US interval was $2,000 \mathrm{msec}$ but the CSA duration was $500 \mathrm{msec}$, thus leaving a trace interval of $1,000 \mathrm{msec}$ between the offset of CSA and the onset of CSB. Each day of serial compound training contained 60 reinforced serial compound trials (CSACSB-US) and 6 unreinforced test trials, 3 each of CSA-alone and CSB-alone. A test trial was administered on every 11 th trial, with the restriction that no two test trials of the same component occur adjacent to one another. In all groups, half the subjects received the tone as CSA and the light as CSB; the other half of the subjects received the reverse assignment of stimuli as CSA and CSB. Throughout training, the average intertrial interval (ITI) was $60 \mathrm{sec}$ (range: $50-70 \mathrm{sec}$ ).

A CR was defined as any extension of the NM exceeding $.5 \mathrm{~mm}$. A CR on serial compound trials was defined as any response initiated after CSA onset but before US onset. A CR on test trials was defined as any response initiated during a 2,000-msec interval af- ter CS onset. Unless otherwise noted, planned contrasts were used to analyze the data and the rejection level was set according to a Type I error rate of .05 .

\section{Results}

During Stage 1, Groups B-OL and B-SQ showed relatively rapid $C R$ acquisition, reaching mean levels of $85 \%$ CRs and $92 \%$ CRs, respectively. Any apparent difference between Groups B-OL and B-SQ failed to reach statistical significance. For Groups R-OL and R-SQ, observations during 500-msec periods corresponding to CSB presentations revealed that the likelihood of a spontaneous response remained at a level less than $1 \%$ throughout Stage 1.

Figure 1 depicts the percent $\mathrm{CRs}$ on serial compound trials, as measured from CSA onset through CSB to US onset in Stage 2, as a function of 2-day blocks. Examination of both panels reveals that Groups B-OL and B-SQ showed levels of responding exceeding $90 \% \mathrm{CRs}$, which represented a continuation of responding established during previous training with CSB. The naive controls, Groups R-OL and R-SQ, showed steady CR acquisition to the serial compound that exceeded $90 \%$ CRs by Days 5-6 of Stage 2 training. By the end of Stage 2, all four groups had attained statistically indistinguishable levels of responding to the compound.

To delineate more precisely the compounded effects of CSA and CSB on responding, measurements were taken during the final 500-msec portion of serial compound trials after the onsets of both CSB and CSA, thus excluding the initial portion in which only CSA or its representation was operative. In calculating the likelihood of a CR's being initiated during the final portion of the serial compound

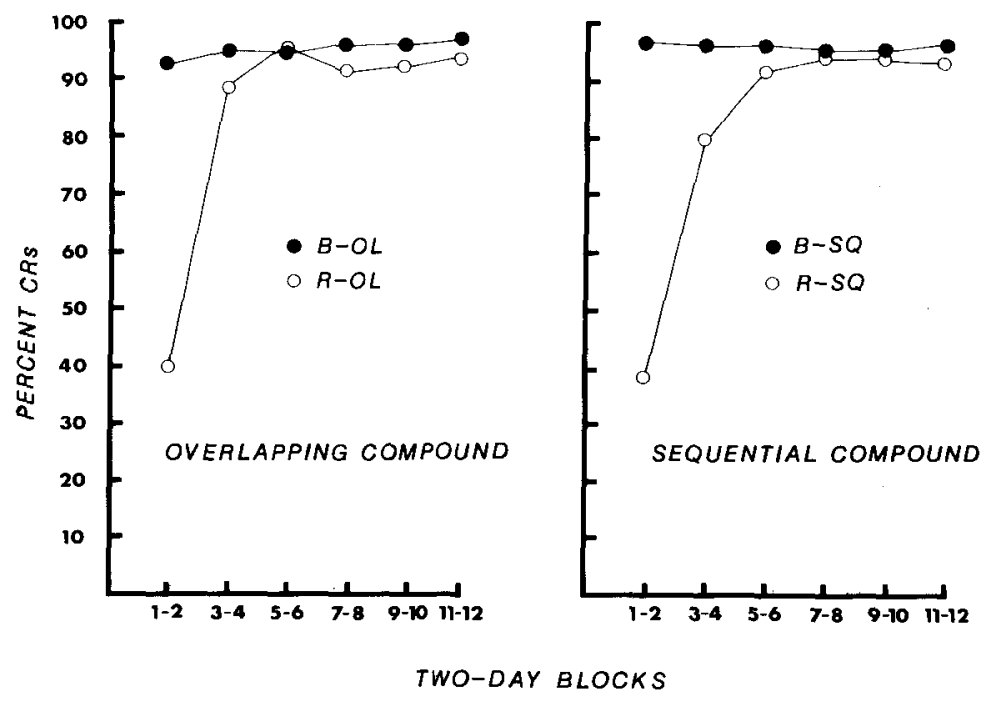

Figure 1. Mean percentage of CRs on serial compound trials as measured from CSA onset through CSB up to US onset as a function of days in Experiment 1. The left-hand panel shows the acquisition curves for overlapping compound groups, namely the CSB-pretrained group (B-OL) and the rest control group (R-OL). The right-hand panel shows the acquisition curves for the corresponding sequential compound groups (B-SQ and R-SQ). 
trials, it was necessary to exclude trials on which a $C R$ had been initiated earlier during either CSA or its trace interval, because a partial or complete closure of the NM often persisted into the CSB period and obscured any additional responses (Kehoe, 1979; Kehoe, MarshallGoodell, \& Gormezano, 1987; Kehoe \& Morrow, 1984). Hence, this measure of CR likelihood was conditional upon the prior absence of a CR during the trial. These measurements of $\mathrm{CRs}$ confirmed the pattern of responding shown in Figure 1. That is to say, Groups B-OL and B-SQ showed levels of responding exceeding $90 \%$ CRs throughout Stage 2, and Groups R-OL and R-SQ showed CR acquisition that exceeded $90 \%$ CRs by Days 5-6 of Stage 2 training.

Figure 2 depicts percent CRs to CSA as measured during the 1,500-msec interval following CSA onset but before CSB onset on serial compound trials. Inspection of the left-hand panel reveals that Groups B-OL and R-OL both displayed an initial rise in responding during CSA to levels between $40 \%$ and $50 \%$ CRs followed by a more gradual decline, a pattern that has been commonly observed in serial compound conditioning with the rabbit (e.g., Kehoe, Gibbs, Garcia, \& Gormezano, 1979; Kehoe \& Morrow, 1984). For the overlapping compound, there was only a slight indication of blocking; Group B-OL showed a level of responding to CSA that was about 8 percentage points lower than that of Group R-OL during Days 3-8 of serial compound training. Examination of the right-hand panel reveals that partial blocking appeared in the sequential compound. Specifically, Group B-SQ's level of responding never exceeded $20 \%$ CRs, whereas the control group, Group R-SQ, displayed an initial rise in responding to CSA that reached $42 \%$ CRs before a decline appeared.

In statistical comparisons, the rise followed by a decline in responding to CSA was confirmed by a significant quadratic trend over days of Stage 2 training $[F(1,28)=$ 5.93]. Statistical evidence for blocking resided in a significant interaction of this quadratic trend over days with the comparison for the blocking groups versus the control groups $[F(1,28)=5.58]$ indicating that Groups B-OL and B-SQ showed flatter performance curves than Groups R-OL and R-SQ. Beyond differences between the blocking and control conditions, the overlapping compound groups generally showed a higher level of responding than did the sequential compound groups $[F(1,28)=4.71]$. Examination of responding on CSA test trials revealed a similar pattern of results but no statistical corroboration.

Figure 3 depicts the percent CRs on CSB test trials in Stage 2. Inspection of the left-hand panel reveals that, for the overlapping compound, Group B-OL showed a pronounced drop in responding from a level of approximately $77 \%$ CRs at the beginning of Stage 2 to a stable level around 53\% CRs. In Group R-OL, responding on CSB test trials was negligible throughout Stage 2, hovering around $5 \%$ CRs. Examination of the right-hand panel reveals that Group B-SQ also showed a decline in responding to CSB from $81 \%$ CRs at the beginning of Stage 2 to a terminal level around $40 \%$ CRs. Group R-SQ showed a modest rise in responding to CSB that reached a stable level around 35\% CRs.

The statistical comparisons confirmed that responding to CSB in the pretrained groups was higher than in the control groups $[F(1,28)=22.40]$. Although the differ-

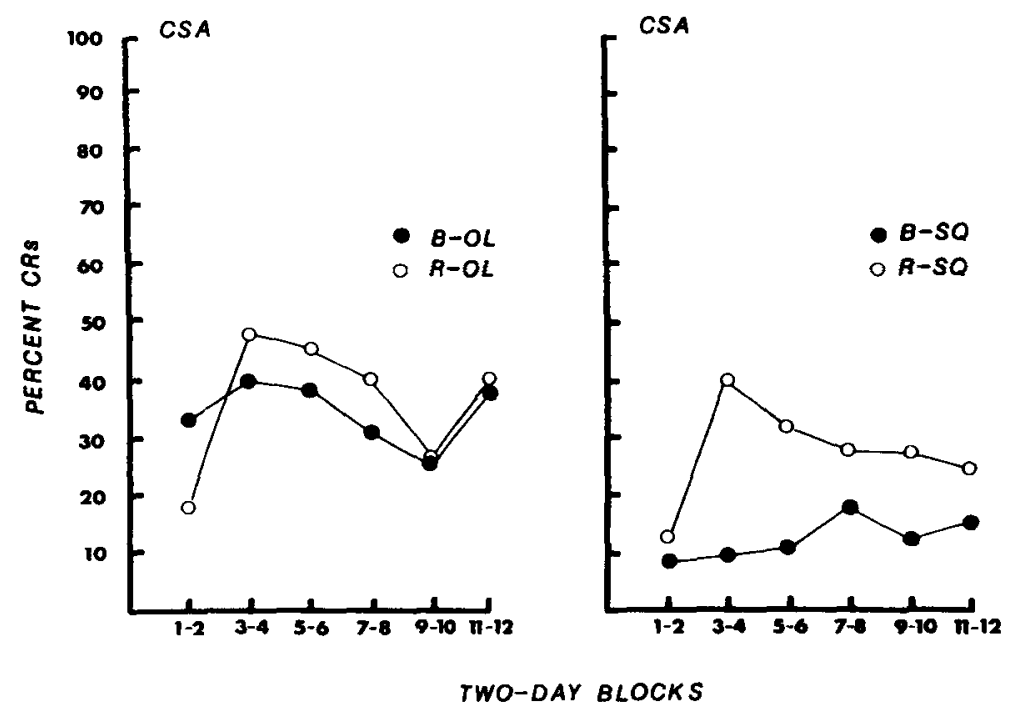

Figure 2. Mean percentage CRs to CSA as measured between CSA onset to CSB onset as a function of days in Experiment 1 . The left-hand panel shows the acquisition curves for overlapping compound groups, namely the CSB-pretrained group (B-OL) and the rest control group (R-OL). The right-hand panel shows the acquisition curves for the corresponding sequential compound groups (B-SQ and R-SQ). 


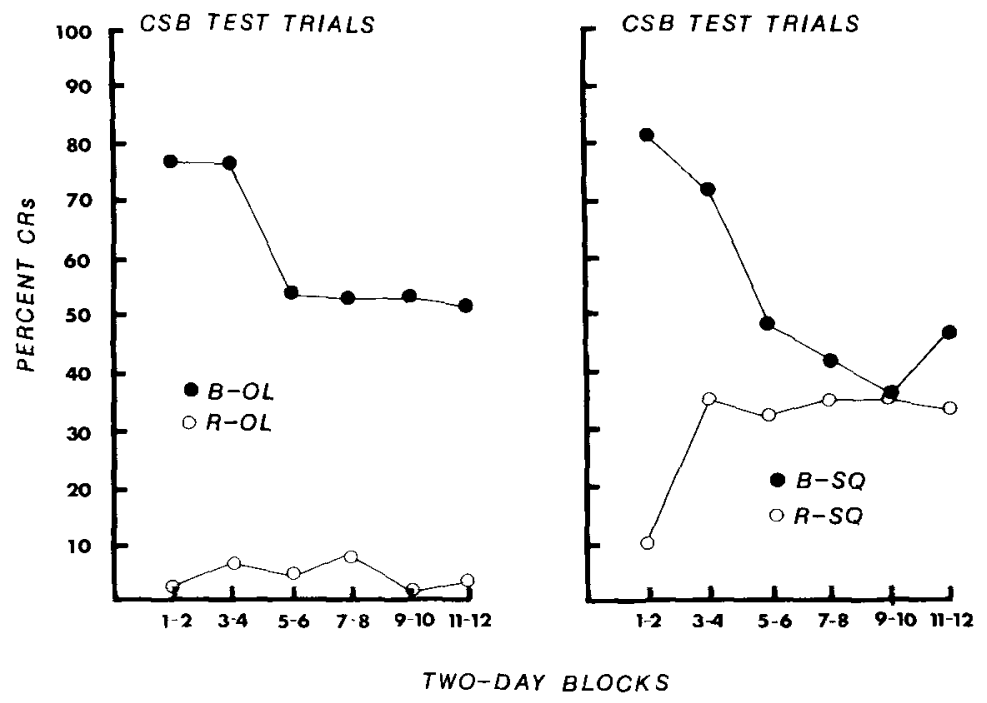

Figure 3. Mean percentage CRs on CSB test trials as a function of days in Experiment 1 . The left-hand panel shows the acquisition curves for overlapping compound groups, namely the CSB-pretrained group (B-OL) and the rest control group (R-OL). The right-hand panel shows the acquisition curves for the corresponding sequential compound groups (B-SQ and R-SQ).

ence between the blocking and control groups was greater in the overlapping compound than in the sequential compound, the appropriate interaction failed to reach significance $[F(1,28)=3.56, p<.10]$. Across Stage 2 training, the linear trends in response levels interacted with the difference between the blocking and control conditions $[F(1,28)=26.27]$. Further comparisons revealed that both Groups B-OL and B-SQ displayed significant downward linear trends in responding to CSB $[F \mathrm{~s}(1,28)$ $=11.85$ and 24.32 , respectively].

\section{Discussion}

The present results revealed that after prior training of CSB, there was (1) modest evidence for blocking of CR acquisition to CSA but (2) considerable evidence for declines in responding on CSB test trials. On the one hand, the weak blocking effect is consistent with previous observations that blocking of CR acquisition to CSA in a serial compound is usually incomplete (e.g., Gibbs, 1979; Kehoe et al., 1981; Kohler \& Ayres, 1982). On the other hand, the declines in responding to the pretrained CSB are a novel outcome of a blocking manipulation but do converge with previous observations of the temporal primacy effect. Moreover, the low level of responding on CSB test trials in the rest control groups matches previous demonstrations of the temporal primacy effect (e.g., Kehoe, 1979; Kehoe et al., 1979). For an unequivocal demonstration of the temporal primacy effect, however, the present experiment would have needed a singlestimulus CSB control in Stage 2. In the absence of such a control, the high level of CR acquisition to CSB in Stage 1 provides some evidence that the serial compound training caused both the low level of responding to CSB in the rest control groups and the decline in responding to CSB in the pretrained groups.

The present results partially confirm predictions generated by the Sutton-Barto model. Most notably, the decline in responding on CSB test trials in the pretrained groups generally agrees with the model. By the same token, however, Barto and Sutton's (1982) simulations predict that the decline in responding to CSB should have been confined to the overlapping compound and should not have appeared in the sequential compound. In defense of the Sutton-Barto model, it can be noted that the predicted differences between overlapping and sequential compounds are based on the simplifying assumption that the effective representation of CSA lasts only as long as the duration of the nominal CS. Instead, in the sequential compound, it appears that the representation of CSA outlasts its 500 -msec duration.

\section{EXPERIMENT 2}

In order to delineate empirically the effective duration of CSA's representation in producing the temporal primacy effect after initial CSB-US training, Experiment 2 examined responding to the sequential compound and its components as a function of the interval between CSA and CSB-US. In previous examinations of the temporal primacy effect, the deficit in CR acquisition to CSB progressively disappeared as the interval between CSA offset and CSB onset was increased up to $2 \mathrm{sec}$ (Kehoe et al., 1979). Accordingly, in the present experiment, all subjects were given initial CSB-US training, followed by 
CSA-CSB-US training with intervals of 0,1 , or $2 \mathrm{sec}$ between the CSA offset and CSB onset. To provide a baseline against which to detect declines in responding to CSB in the serial compound groups, the present experiment included a single-stimulus group given CSB-US pairings throughout both stages of training.

\section{Method}

Subjects. The subjects were 32 naive female albino rabbits, 70-80 days old and weighing approximately $1.5 \mathrm{~kg}$ on arrival.

Apparatus and Procedure. Unless otherwise noted, the apparatus and procedure were identical to those used in Experiment 1. All rabbits received 1 day of preparation, 2 days of recovery, 1 day of adaptation, 4 or 6 days in Stage 1, with CSB-US training, and 12 days in Stage 2, which entailed either CSB-US training or serial compound training. Following adaptation, the animals were assigned randomly to one of four groups $(n=8)$ that were labeled $\mathrm{BB}, \mathrm{AOB}$, $A 1 B$, and $A 2 B$. All four groups were initially given acquisition training in which each day contained $60 \mathrm{CSB}$-US pairings for which the CSB-US interval was $500 \mathrm{msec}$. The animals that received tone as CSB were given 4 days of Stage 1 training, and the animals that received light as CSB were given 6 days of Stage 1 training. During Stage 2 training, Group BB continued to receive CSB-US pairings. The remaining three groups were trained with a sequential compound in which the interval between CSA and CSB was manipulated. Specifically, there was a $0-, 1-$, and $2-\sec$ interval between CSA offset and CSB onset in Groups A0B, A1B, and A2B, respectively. The CS duration was $500 \mathrm{msec}$ for both CSA and CSB. Both tone and light stimuli were used as the CSs and were counterbalanced within each group. During serial compound training, all groups received 60 reinforced trials per day, which were interspersed with 3 unreinforced tests each for CSA and CSB. Each block of 11 trials contained 10 compound trials with the 11 th trial alternating randomly between a test of either CSA or CSB. The mean ITI was $60 \mathrm{sec}$ (range: 50-70 sec).

\section{Results and Discussion}

During Stage 1, Groups BB, A0B, A1B, and A2B showed relatively rapid CR acquisition to CSB, reaching mean terminal levels of $93 \%, 75 \%, 83 \%$, and $90 \% \mathrm{CRs}$, respectively. Any apparent differences among response levels to CSB failed to reach statistical significance.

In Stage 2, Group BB continued to respond to CSB at levels in excess of $90 \%$ CRs. For the serial compound groups, the addition of CSA did not appear to alter responding on reinforced trials. For Groups A0B, A1B, and $A 2 B$, percent $C R$ was calculated (1) for the serial compound as a whole, from CSA onset through CSB to US onset, and (2) for the final 500-msec portion of the compound that contained CSB. For responding to the serial compound as a whole and during its CSB portion, Groups A1B and A2B responded at levels above $90 \%$ CRs throughout training. Group A0B initially responded at a lower level of $75 \% \mathrm{CRs}$, which gradually increased across sessions to terminate at $92 \%$ CRs. Statistical comparisons revealed a significant linear trend across Groups A0B, $A 1 B$, and $A 2 B$ in their response levels during the compound as a whole $[F(1,28)=9.78]$ and the CSB portion $[F(1,28)=11.18]$. The convergence of Group $\mathrm{AOB}$ with Groups A1B and A2B was confirmed by a significant interaction of the trace interval (linear trend) $x$ days (linear trend) for both response measures, $\left[F_{\mathrm{s}}(1,28)=\right.$ 4.33 and 7.52].

As was observed in Experiment 1, the added CSA commanded relatively little responding during itself or its trace interval. On the first day of Stage 2 training, Groups A0B, $\mathrm{A} 1 \mathrm{~B}$, and $\mathrm{A} 2 \mathrm{~B}$ responded uniformly to CSA at levels around $25 \%$ CRs. Subsequently, Groups A1B and A2B gradually increased their responding to CSA to reach terminal levels of $37 \%$ and $32 \%$ CRs, respectively. In contrast, Group A0B showed gradual decreases in responding to CSA across days to terminate at 5\% CRs. However, statistical comparisons of response levels to CSA across groups failed to attain statistical significance.

Figure 4 shows percent CRs on CSB test trials as a function of 2-day blocks. Inspection of Figure 4 reveals that response levels in Groups A0B, A1B, and A2B gradually decreased across sessions, while responding in Group BB remained relatively stable, at a level greater than $90 \%$ CRs. Statistical comparisons revealed that the main-effect comparison of Group BB versus the serial compound groups was significant $[F(1,28)=17.98]$. Furthermore, the decline in responding to CSB across days was confirmed by a significant interaction of the groups comparison (Group BB vs. serial compound groups) $\times$ days (linear trend) $[F(1,28)=6.65]$. Although Groups A0B, A1B, and A2B all showed a decrease in CR likelihood on CSB test trials, the magnitude of the decline was inversely related to the trace interval between CSA and CSB. That is to say, Group A0B showed a rapid decline from its mean level of $75 \% \mathrm{CRs}$ at the end of Stage 1 to $44 \%$ CRs over the first 2 days of Stage 2 serial com-

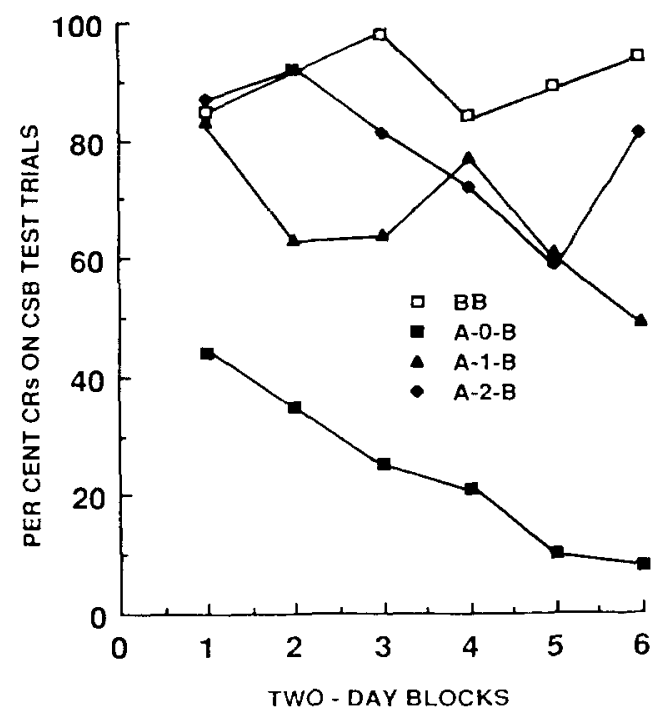

Figure 4. Mean percentage CRs on CSB test trials as a function of days in Experiment 2. Separate curves are shown for the singlestimulus CSB control group (BB) and for the sequential compound groups $(\mathrm{AOB}, \mathrm{A1B}$, and $\mathrm{A2B})$ that received training in which there was $0,1,000$, or 2,000 msec between the offset of CSA and the onset of CSB. 
pound training. Thereafter, Group A0B showed a steady decline to a mean level of $8 \%$ CRs at the end of Stage 2 training. Groups A1B and A2B showed progressively smaller declines, terminating at $49 \%$ and $81 \% \mathrm{CRs}$, respectively. Statistical comparisons confirmed this inverse relationship between the decline in responding on CSB test trials and the trace interval in terms of a significant linear trend in the means of Groups A0B, A1B, and A2B, which were $24 \%, 66 \%$, and $79 \%$ CRs, respectively $[F(1,28)=30.98]$.

Part of the low level of responding on CSB test trials in Group AOB can be attributed to its lower level of CR acquisition to CSB during Stage 1 training. To determine whether this lower initial level of acquisition contributed to the apparent decline in responding to CSB during Stage 2, Group AOB's data were reexamined after excluding subjects that had displayed low levels of CR acquisition during Stage 1. Specifically, 3 subjects entered Stage 2 with response levels around $50 \% \mathrm{CRs}$; the remaining 5 subjects had response levels in excess of $90 \%$ CRs. Among those 5 subjects, there was still a pronounced decline in responding to CSB from $92 \%$ CRs at the end of Stage 1 to $60 \%$ CRs on the first 2 days of Stage 2 training, which further declined and terminated at $13 \% \mathrm{CRs}$.

The pattern of responding on CSB test trials across trace intervals indicates that the representation of CSA persists, but in a decaying form, for periods up to $2,000 \mathrm{msec}$. This estimate of the time course of CSA's representation closely parallels estimates obtained previously with the rabbit NM preparation. Specifically, the present estimate is virtually identical to that obtained by Kehoe et al. (1979), who examined the temporal primacy effect in sequential compounds for intervals of $0,500,1,000$, and $2,000 \mathrm{msec}$ between CSA offset and CSB onset. Likewise, second-order conditioning shows a similar decay function over intervals between 0 and 2,000 msec between the offset of the second-order CS and the onset of the firstorder CS (Gormezano \& Kehoe, 1981, p. 23). More generally, examinations of trace conditioning reveal that the rate and asymptote of CR acquisition decrease as CS-US intervals increase from 200 to $2,000 \mathrm{msec}$ (Kehoe et al., 1979, Experiment 2; Schneiderman, 1966; Smith, 1968). Despite the close agreement among these converging operations, it does not appear that the representation of a brief CS entirely disappears within 2,000 msec after its offset. Recently, there has been evidence that a residual representation of CSA in a sequential compound survives in a discriminable form for periods up to $12,000 \mathrm{msec}$. Kehoe et al. (1987) examined a conditional discrimination entailing two sequential compounds, namely a tonelight sequence and a noise-light sequence, one of which was paired with the US $(A-X+)$ while the other was presented alone $(B-X-)$. Even when the interval between the initial stimulus ( $A$ or $B$ ) and the shared common stimulus $(X)$ was increased to $11,400 \mathrm{msec}$, the subjects still showed some differential responding during $X$ as a function of whether it was preceded by $A$ or $B$.
Notwithstanding uncertainty about the exact duration of a CS representation, it is clearly inaccurate to identify the duration of a nominal CS with the duration of its effective representation. With regard to the Sutton-Barto model, there is no difficulty in altering its assumptions regarding the time-course of a CS representation. In fact, Moore and his associates, in collaboration with Sutton and Barto, have proposed at least two schemes for more accurately describing the time course of a CS representation (Blazis, Desmond, Moore, \& Berthier, 1986; Desmond, Blazis, Moore, \& Berthier, 1986; Moore et al., 1985).

\section{GENERAL DISCUSSION}

The present experiments provide further evidence that the temporal primacy of CSA relative to CSB in a serial compound can apparently override otherwise very potent conditioning variables, namely contiguity, salience, and prior training. In the basic demonstrations of the temporal primacy effect, training with a reinforced serial compound (CSA-CSB-US) has impaired response acquisition to CSB, even though CSB is more contiguous than CSA to the US (e.g., Egger \& Miller, 1962; Kehoe, 1979; Kehoe et al., 1979; Wickens, 1959, 1965, 1973). Furthermore, these impairments have been observed even when CSB was a highly salient tone stimulus that, in a simultaneous compound, had overshadowed the light stimulus used as CSA (Kehoe, 1983). The present experiments demonstrated that responding to CSB can suffer large deficits even though CSB had undergone prior training, had acquired a considerable CR-evoking capacity, and showed some ability to block CR acquisition to CSA.

The present findings also add to the seemingly paradoxical evidence that the deficits in responding on CSB test trials represent something other than a failure of CSB to gain associative strength in serial compound training. Previous findings have indicated that the impairment of responding seen when CSB is tested by itself does not seem to appear inside the context of the compound. First, considerable responding during CSB has been observed when assessed inside the context of the serial compound (Gaioni, 1982; Kehoe, 1979; Seger \& Scheuer, 1977). Similarly, in the present experiments, responding during the serial compound occurred largely during CSB. At the end of serial compound training, the likelihood of a CR between the onset of CSA and the onset of CSB was $40 \%$ CRs or less, but CR likelihood during CSB exceeded $90 \%$ CRs. Second, the conditioning parameters of CSB have considerable influence on $\mathrm{CR}$ acquisition in all portions of a serial compound. Specifically, the CSB-US interval and CSB intensity determine the rate and asymptote of CR acquisition (Kehoe, 1979, 1983).

The theoretical implications of the present findings provide support for the Sutton-Barto model and, conversely, seem to require the incorporation of real-time processes in alternative accounts of stimulus selection phenomena. 
In broad terms, theories of stimulus selection can be divided into "perceptual" and "distributive" hypotheses (James \& Wagner, 1980). Distributive theories can be further subdivided into associative competition models (e.g., Rescorla \& Wagner, 1972), selective attention models (e.g., Mackintosh \& Reese, 1979), and dynamic attention models (e.g., Mackintosh, 1975; Pearce \& Hall, 1980).

\section{Perceptual Hypotheses}

Perceptual hypotheses presuppose that a constant, nominal CS produces different neural encodings, depending on whether or not the CS is presented in the context of other stimuli. By assuming that different encodings of CSB occur inside versus outside the context of CSA, the perceptual hypotheses can readily explain the disparity between the effectiveness of CSB inside a serial compound and its inability to evoke CRs outside a serial compound (Borgealt, Donahoe, \& Weinstein, 1972; Hancock, 1982; Kehoe, 1979, 1983). Specifically, CSB and its conditioning parameters, as encoded inside the context of CSA, would have full access to the associative apparatus. However, the associative strength of CSB inside the context of CSA would generalize only weakly to tests of CSB outside the context of CSA. With respect to CSA, only responding to the later portions of CSA could possibly suffer a generalization decrement, because the initial portion of CSA is always presented and presumably encoded in the context of the background apparatus stimuli.

The perceptual hypotheses have considerable difficulty in explaining the levels of responding to both CSA and CSB in the blocking groups. The decline in responding on CSB test trials cannot be attributed to a generalization decrement. The encoding of CSB outside the context of CSA would have acquired its own independent associative strength as a consequence of initial CSB-US training. Thus, serial compound training should have been irrelevant to responding on CSB test trials. By the same token, even partial blocking of CR acquisition to CSA cannot be attributed to a generalization decrement, because the initial portion of CSA prior to CSB always occurs within the same context, that of the background apparatus stimuli in both the blocking and control conditions (Kehoe, 1982).

\section{Distributive Hypotheses}

Distributive hypotheses contend that two compounded stimuli will compete for a fixed amount of "associative strength" (e.g., Rescorla \& Wagner, 1972) or "attention" (Sutherland \& Mackintosh, 1971, p. 122). Consequently, blocking occurs because prior training with one component permits it to capture all the available associative strength or attention, thus preventing an added component from gaining access to the associative apparatus. More recently, dynamic attention theories have loosened the assumption that there is a direct competition between stimuli for a fixed resource. Nevertheless, dynamic at- tention theories propose that there is a partial tradeoff among the learning-rate parameters of the components of a compound (e.g., Mackintosh, 1975; Moore \& Stickney, 1980; Pearce \& Hall, 1980).

Without the addition of real-time processes, the distributive models cannot readily explain the decline in responding to CSB. According to Rescorla and Wagner's (1972) model, there should have been maintenance of responding to CSB accompanied by profound blocking of CSA because CSB had captured all the available associative strength. The selective attention and dynamic attention models have more latitude in their predictions regarding CSA but not CSB. The selective attention models would expect CSB to retain the associative strength gained in Stage 1 even if CSA did capture some attention prior to the onset of CSB during serial compound training (Kehoe et al., 1981; Mackintosh \& Reese, 1979). Likewise, the dynamic-attention models would expect the learning-rate parameter of CSA to remain high because CSA predicts CSB. Conversely, because CSB is well predicted by CSA, CSB might suffer a decline in its learning rate during serial compound training. Nevertheless, the associative strength acquired by CSB during initial training should remain intact.

\section{Real-Time Models}

With the addition of real-time processes, the distributive hypotheses can provide a coherent account for many of the seemingly paradoxical features of serial compound conditioning. In particular, the insights concerning the role of CSA-CSB relations contained in the Sutton-Barto model might be extended to other distributive processes. As detailed in the introduction, the Sutton-Barto model contends that the temporal primacy effect arises from (1) second-order conditioning of CSA at the point of CSB onset, followed by (2) competition between CSA and CSB at the point of US onset. Thus, the temporal primacy of CSA does not override the conditioning parameters of CSB; rather, CSA feeds on the conditioning parameters of CSB, namely its contiguity with the US, its salience, and/or its prior training. Anything that contributes to the associative strength of CSB will ultimately contribute to the associative strength of CSA and, accordingly, the temporal primacy effect. Consequently, the Sutton-Barto model would expect the conditioning parameters of CSB to determine the rate of $\mathrm{CR}$ acquisition in all portions of a serial compound.

From the perspective of the Sutton-Barto model, the only puzzling features of the present findings were the relatively low level of responding to CSA in all serial compound groups and the evidence for partial blocking of CSA in the pretrained groups. Although blocking of CR acquisition to CSA was modest in Experiment 1, there are now enough demonstrations of partial blocking of CSA to indicate that it is a reliable outcome (see Kehoe, 1982, p. 42; Kohler \& Ayres, 1982). Contrary to the results, the Sutton-Barto model generally predicts that impair- 
ments or decreases in responding to CSB should be accompanied by unimpeded CR acquisition to CSA. Furthermore, the Sutton-Barto model would predict blocking of CSA only if it were simultaneous with CSB.

The generally low level of responding to CSA may arise from stimulus processing factors other than the associative processes described by the Sutton-Barto model. In comparison with other studies of serial compound conditioning with the rabbit NM (e.g., Gibbs, 1979; Kehoe et al., 1979; Kehoe \& Morrow, 1984), the level of responding to CSA was approximately 25 percentage points lower than might have been expected. In the case of Group R-SQ in Experiment 1, the conditioning parameters were very similar to those used in studies by Kehoe et al. (1979, Experiments 1 and 3) and Gibbs (1979). The only notable difference was that the 500 -msec duration of both CSA and CSB in the present serial compounds was slightly longer than the $400-\mathrm{msec}$ duration of CSA and CSB used in previous studies.

Although the difference in CS durations may seem of little significance, recent studies with simultaneous compound stimuli have indicated that the engagement of a gestalt-like unique stimulus depends crucially on the duration of the CSs (Kehoe, 1986; Kehoe \& Graham, in press; Kehoe \& Schreurs, 1986). Specifically, 300-msec CSs only minimally engage a unique stimulus, and the representations of the individual CSs compete with each other for associative strength. In contrast, 800-msec CSs almost fully engage a unique stimulus, and this separate representation of the compound almost fully overshadows those of the components. Thus, an increase in CSA and CSB duration from 400 to $500 \mathrm{msec}$ may have produced a dramatic increase in the engagement of the unique stimulus in the final portion of the serial compound in which the representation of CSA would overlap that of CSB. If so, responding would tend to be concentrated during the period of the unique stimulus. There is nothing to prevent a unique-stimulus hypothesis from being appended to the Sutton-Barto model in the same way as a uniquestimulus hypothesis has been appended to the RescorlaWagner model (Kehoe \& Gormezano, 1980). However, the ramifications of the unique-stimulus hypothesis for the functioning of the Sutton-Barto model would depend crucially on assumptions regarding the temporal relations between the unique stimulus and the separate representations of CSA and CSB.

The failure of the Sutton-Barto model to predict blocking of CSA by CSB in a serial compound may arise from one of the model's simplifying assumptions. Specifically, the effective learning rate is a product of the real-time CS trace variable $[x i(t)]$ and a fixed parameter $(c)$. As may be apparent, the Sutton-Barto model in its present form does not assume that the learning rate is determined by the intensity of the reinforcing stimulus. Thus, when trace variables are equated, the learning rate for CSA at CSB onset would be the same as that for CSB at US onset. If the $c$ parameter were anchored to the intensity of the reinforcing stimulus, then the rate of second-order conditioning of CSA at CSB onset could be reduced relative to first-order conditioning of CSB at US onset. This reduced rate of second-order conditioning of CSA would slow down the emergence of the temporal primacy effect, permitting VB to remain high and, consequently, precluding VA from gaining large increments in associative strength at US onset, at least on a temporary basis.

If adjustments in the learning rates are not sufficient to explain the joint appearance of the temporal primacy effect and the blocking of CSA, there are more radical departures from the original Sutton-Barto model that could still be accommodated within its framework. For example, it is possible to incorporate a dynamic attention rule while retaining the axioms of the Rescorla-Wagner model (cf. Frey \& Sears, 1978; Wagner, 1978). Under such a revision, initial training would ensure that CSB would gain a high learning-rate parameter as well as a high associative strength. Subsequently, during serial compound conditioning, the high predictive value of CSB for the US would retard increases in CSA's learning-rate parameter. Although CSA would still gain associative strength from CSB, CSA's slow rate of CR acquisition might well fall behind that of a rest control group in which CSA and CSB would start out in all respects on a more even footing.

\section{REFERENCES}

Barto, A. G., \& Sutton, R. S. (1982). Simulation of anticipatory responses in classical conditioning by a neuron-like adaptive element. Behavioral Brain Research, 4, 221-235.

Blazis, D. E. J., Desmond, J. E., Moore, J. W., \& Berthier, N. E (1986). Simulation of the classically conditioned nictitating membrane response by a neuron-like adaptive element: A real-time variant of the Sutton-Barto model. In C. Clifton (Ed.), Proceedings of the Eighth Annual Conference of the Cognitive Science Society (pp. 154-175) Hillsdale, NJ: Erlbaum.

Borgealt, A. J., Donahoe, J. W., \& Weinstein, A. (1972). Effects of delayed and trace components of a compound CS on conditioned suppression and heart rate. Psychonomic Science, 26, 13-15.

Cheatle, M. D., \& Rudy, J. W. (1978). Analysis of second-order odoraversion conditioning in neonatal rats: Implications for Kamin's blocking effect. Journal of Experimental Psychology: Animal Behavior Processes, 4, 237-249.

Desmond, J. E. , Blazis, D. E. J., Moore, J. W., \& Berthier, N. E. (1986). Computer simulations of a classically conditioned response using neuron-like adaptive elements: Response topography. Society for Neuroscience Abstracts, 12, 516.

Egger, D. M., \& Miller, N. E. (1962). Secondary reinforcement in rats as a function of information value and reliability of the stimulus. Journal of Experimental Psychology, 64, 97-104.

FREY, P. W., \& SEARS, R. J. (1978). Model of conditioning incorporating the Rescorla-Wagner associative axiom, a dynamic attention process, and a catastrophe rule. Psychological Review, 85, 321-348.

GaIONI, S. J. (1982). Blocking and non-simultaneous compounds: Comparisons of responding during compound conditioning and testing. Pavlovian Journal of Biological Sciences, 17, 16-29.

GiBBS, C. M. (1979). Serial compound classical conditioning (CS1-CS2$U C S)$ : Effects of CS2 intensity and pretraining on component acquisition. Unpublished doctoral dissertation, University of Iowa.

Gormezano, I. (1966). Classical conditioning. In J. B. Sidowski (Ed.), Experimental methods and instrumentation in psychology (pp. 385420). New York: McGraw-Hill. 
Gormezano, I., \& KeHOE, E. J. (1981). Classical conditioning and the law of contiguity. In P. Harzem \& M. D. Zeiler (Eds.), Advances in analysis of behaviour (Vol. 2, pp. 1-45). Sussex: Wiley.

HANCOCK, R. A., JR. (1982). Tests of the conditioned reinforcement value of sequential stimuli in pigeons. Animal Learning \& Behavior, 10, 46-54.

Holland, P. C. (1980). Second-order conditioning with and without unconditioned stimulus presentation. Joumal of Experimental Psychology: Animal Behavior Processes, 6, 238-250.

JAMES, J. H., \& W AGNER, A. R. (1980). One-trial overshadowing: Evidence of distributive processing. Journal of Experimental Psychology: Animal Behavior Processes, 6, 188-205.

KeHOE, E. J. (1979). The role of CS-US contiguity in classical conditioning of the rabbit's nictitating membrane response to serial stimuli. Learning \& Motivation, 10, 23-38.

KEHOE, E. J. (1982). Conditioning with serial compound stimuli: Theoretical and empirical issues. Experimental Animal Behaviour, 1, 30-65.

KенOE, E. J. (1983). CS-US contiguity and CS intensity in conditioning of the rabbit's nictitating membrane response to serial and simultaneous compound stimuli. Joumal of Experimental Psychology: Animal Behavior Processes, 9, 307-319.

KEHOE, E. J. (1986). Summation and configuration in conditioning of the rabbit's nictitating membrane response to compound stimuli. Journal of Experimental Psychology: Animal Behavior Processes, 12, 186-195.

Kehoe, E. J., Gibbs, C. M., Garcia, E., \& Gormezano, I. (1979). Associative transfer and stimulus selection in classical conditioning of the rabbit's nictitating membrane response to serial compound CSs. Journal of Experimental Psychology: Animal Behavior Processes, 5 , 1-18.

Kehoe, E. J., \& Gormezano, I. (1980). Configuration and combination laws in conditioning with compound stimuli. Psychological Bulletin, 87, 351-378.

Kehoe, E. J., \& Graham, P. (in press). Summation and configuration Stimulus compounding and negative patterning in the rabbit. Journal of Experimental Psychology: Animal Behavior Processes.

Kehoe, E. J., Marshall-Goodell, B. S., \& Gormezano, I. (1987). Differential conditioning of the rabbit's nictitating membrane response to serial compound stimuli. Journal of Experimental Psychology: Animal Behavior Processes, 13, 17-30.

Kehoe, E. J., \& Morrow, L. D. (1984). Temporal dynamics of the rabbit's nictitating membrane response in serial compound conditioned stimuli. Joumal of Experimental Psychology: Animal Behavior Processes, 10, 205-220.

KehoE, E. J., \& Schreurs, B. G. (1986). Compound-component differentiation as a function of CS-US interval and CS duration in the rabbit's nictitating membrane response. Animal Learning \& Behavior, 14, 144-154.

Kehoe, E. J., Schreurs, B. G., \& AMOdeI, N. (1981). Blocking acquisition of the rabbit's nictitating membrane response to serial conditioned stimuli. Learning \& Motivation, 12, 92-108.

KOHLER, E. A., AYRES, J. J. B. (1982). Blocking with serial and simultaneous compounds in a trace conditioning procedure. Animal Learning \& Behavior, 10, 277-287.

MACKInTosh, N. J. (1975). A theory of attention: Variation in the associability of stimuli with reinforcement. Psychological Review, 82, 276-298.
Mackintosh, N. J., \& Reese, B. (1979). One-trial overshadowing. Quarterly Journal of Experimental Psychology, 31, 519-526.

Moore, J. W., Desmond, J. E., Berthier, N. E., Blazis, D. E. J., SutTon, R. S., \& BARTo, A. G. (1985). Connectionistic learning in real-time: Sutton-Barto adaptive element and classical conditioning of the rabbit nictitating membrane response. Proceedings of the Seventh Annual Conference of the Cognitive Science Society, August 15-17, Irvine, CA.

MOORE, J. W., STICKNEY, K. J. (1980). Formation of attentionalassociative networks in real time: Role of the hippocampus and implications for conditioning. Physiological Psychology, 8, 207-217.

Pearce, J. M., \& Hall, G. (1980). A model for Pavlovian conditioning: Variations in the effectiveness of conditioned but not of unconditioned stimuli. Psychological Review, 87, 532-552.

Pearce, J. M., Nicholas, D. J., \& Dickinson, A. (1981). The potentiation effect during serial compound conditioning. Quarterly Journal of Experimental Psychology, 33B, 159-179.

Rescorla, R. A., \& Wagner, A. R. (1972). A theory of Pavlovian conditioning: Variations in the effectiveness of reinforcement and nonreinforcement. In A. H. Black \& W. F. Prokasy (Eds.), Classical conditioning II (pp. 64-99). New York: Appleton-Century-Crofts.

Revusky, S. H. (1971). The role of interference in association over a delay. In W. K. Honig \& P. H. R. James (Eds.), Animal memory (pp. 155-213). New York: Academic Press.

SCANDRETT, J., \& GoRmezANo, I. (1980). Microprocessor control and A-D data acquisition in classical conditioning. Behavior Research Methods \& Instrumentation, 12, 120-125.

SCHNEIDERMAN, N. (1966). Interstimulus interval function of the nictitating membrane response under delay versus trace conditioning. Journal of Comparative \& Physiological Psychology, 62, 397-402.

SEger, K. A., \& SCHEuer, C. (1977). The informational properties of S1, S2, and the S1-S2 sequence on conditioned suppression. Animal Learning \& Behavior, 5, 39-41.

SMITH, M. C. (1968). CS-US interval and US intensity in classical conditioning of the rabbit's nictitating membrane response. Journal of Comparative \& Physiological Psychology, 66, 679-687.

Sutherland, N. S., \& Mackintosh, N. J. (1971). Mechanisms of animal discrimination learning. New York: Academic Press.

SutTon, R. S., \& BARTo, A. G. (1981). Toward a modern theory of adaptive networks: Expectation and prediction. Psychological Review, 88, 135-171.

WAGNER, A. R. (1978). Expectancies and the priming of STM. In S. H. Hulse, H. Fowler, \& W. K. Honig (Eds.), Cognitive processes in animal behavior (pp. 177-209). Hillsdale, NJ: Erlbaum.

Wickens, D. D. (1959). Conditioning to complex stimuli. American Psychologist, 14, 180-188.

WICKENS, D. D. (1965). Compound conditioning in humans and cats. In W. F. Prokasy (Ed.), Classical conditioning (pp. 323-339). New York: Appleton-Century-Crofts.

WiCKENS, D. D. (1973). Classical conditioning, as it contributes to the analyses of some basic psychological processes. In F. J. McGuigan \& D. B. Lumsden (Eds.), Contemporary approaches to conditioning and learning (pp. 213-243). New York: Winston.

(Manuscript received December 5, 1986; revision accepted for publication May 1, 1987.) 